\title{
SARS-CoV-2 Omicron: evasion of potent humoral responses and resistance to clinical immunotherapeutics relative to viral variants of concern
}

\author{
Anupriya Aggarwal \\ UNSW Sydney \\ Alberto Stella \\ Kirby Institute UNSW \\ Gregory Walker \\ Serology and Virology Division (SAViD), NSW Health Pathology, Randwick, Australia. \\ Anouschka Akerman \\ Kirby Institute UNSW \\ Vanessa Milogiannakis \\ Kirby Institute UNSW \\ Fabienne Brilot \\ The Children's Hospital at Westmead
}

Supavadee Amatayakul-Chantler

CSL Behring, Broadmeadows, Victoria https://orcid.org/0000-0003-3510-7476

\section{Nathan Roth}

CSL Behring AG, Bern, Switzerland

\section{Germano Coppola}

CSL Behring, Broadmeadows, Victoria

\section{Peter Schofield}

Garvan Institute

Jennifer Jackson

Garvan Institute

Helen Lenthall

Garvan Institute

Ohan Mazigi

Garvan Institute

David Langley

Garvan Institute

\section{Yonghui Lu}

Serology and Virology Division (SAViD), NSW Health Pathology, Randwick 
Charles Forster

Serology and Virology Division (SAViD), NSW Health Pathology, Randwick

\section{Samantha McAllery}

Kirby Institute UNSW

Vennila Mathivanan

Kirby Institute UNSW

Christina Fitcher

Kirby Institute UNSW

\section{Alexandra Carey Hoppe}

Kirby Institute UNSW

C. Mee Ling Munier

Kirby Institute UNSW Sydney https://orcid.org/0000-0002-6419-142X

\section{Hans-Martin Jack}

University of Erlangen-Nürnberg, Erlangen, Germany https://orcid.org/0000-0002-6332-8463

\section{Deborah Cromer}

Kirby Institute UNSW

\section{David Darley}

St Vincent's Hospital, Sydney https://orcid.org/0000-0002-9721-101X

\section{Gail Matthews}

St Vincent's Hospital, Sydney

\section{Daniel Christ}

Garvan Institute

\section{David Khoury}

Kirby Institute, University of New South Wales

\section{Miles Davenport}

Kirby Institute, University of New South Wales

\section{William Rawlinson}

Prince of Wales Hospital

\section{Anthony Kelleher}

Kirby Institute UNSW

Stuart Turville ( $\square$ sturville@kirby.unsw.edu.au )

Kirby Institute UNSW Sydney https://orcid.org/0000-0003-1918-5343

\section{Letter}

Keywords:

Posted Date: January 11th, 2022

DOl: https://doi.org/10.21203/rs.3.rs-1207364/v1 
License: (c) (i) This work is licensed under a Creative Commons Attribution 4.0 International License. Read Full License 


\section{Abstract}

Genetically distinct viral variants of severe acute respiratory syndrome coronavirus 2 (SARS-CoV-2) have been recorded since January 2020. Over this time global vaccine programs have been introduced, contributing to lowered COVID-19 hospitalisation and mortality rates, particularly in the first world. In late 2021, the Omicron (B.1.1.529) virus variant emerged, with significant genetic differences and clinical effects from other variants of concern (VOC). This variant a demonstrated higher number of polymorphisms in the gene encoding the Spike (S) protein, and there has been displacement of the dominant Delta variant. We assessed the impact of Omicron infection on the ability of: serum from vaccinated and/or previously infected individuals; concentrated human IgG from plasma donors, and licensed monoclonal antibody therapies to neutralise the virus in vitro. There was a 17 to 27 -fold reduction in neutralisation titres across all donors who had a detectable neutralising antibody titre to the Omicron variant. Concentrated pooled human IgG from convalescent and vaccinated donors had greater breadth of neutralisation, although the potency was still reduced 16-fold. Of all therapeutic antibodies tested, significant neutralisation of the Omicron variant was only observed for Sotrovimab, with other monoclonal antibodies unable to neutralise Omicron in vitro. These results have implications for ongoing therapy of individuals infected with the Omicron variant.

\section{Introduction}

At the beginning of November 2021, the VOC Delta represented over $98 \%$ of SARS-CoV-2 infections sequenced worldwide. Omicron was first identified in South Africa on November $9^{\text {th }}, 2021$ and has primarily driven the fourth viral wave of infection in South Africa. The gene encoding the Spike (S) protein of the Omicron variant typically has 30 amino acid polymorphisms, three deletions and one insertion. Whilst the number of substitutions far exceeds that of other VOCs, the number of changes alone is not an effective surrogate to predict virus fitness in relation to transmission or ability to evade a humoral response generated through prior infection and/or vaccination. From the study of current VOCs and other variants, Omicron unfortunately has critical changes in Spike. These have three main functional effects. Firstly, Omicron has multiple changes within the receptor-binding domain (RBD) associated with antibody evasion and also Angiotensin Converting Enzyme 2 (ACE2) binding affinity. This includes previously described polymorphisms known for their ability to evade antibodies, including those at positions 484 and $477^{1}$. Secondly, many changes in the RBD are associated with potential significant increases in ACE2 affinity. S477N, Q498R and N501Y are located at the extremes of the RBD-ACE2 interface and act to stabilise this interaction ${ }^{2}$. Finally, Omicron shares changes $(\mathrm{H} 655 \mathrm{Y}, \mathrm{N} 679 \mathrm{~K} \& \mathrm{P} 681 \mathrm{H})$ at and around the $\mathrm{S} 1 / \mathrm{S} 2$ furin cleavage site.

Herein we used a rapid and sensitive platform that was developed specifically for the isolation and characterisation of SARS-CoV-2 variants with respect to their relative transmission threat in previously infected and vaccinated populations. Within a week of obtaining the first positive Omicron nasopharyngeal swab sample in Australia, we documented greater resistance to neutralisation of Omicron compared to VOC Beta, Gamma and Delta across serum from both ChAdOx $1 \mathrm{nCoV}-19$ and 
BNT162b2 double dose vaccinated donors (Supplementary Table S1). Further quantification of immune evasion by Omicron was established using four complementary approaches. The first was using individual serum samples from patients recruited to ADAPT, a community-based cohort ${ }^{3}$ of approximately 200 patients followed from the time of diagnosis during all three waves of infection in Australia. We studied a set of 50 samples with the highest humoral responses during convalescence following early viral clade infections in 2020: Clade A and B in March 2020 and Clade 20F in August 2020 and then Delta from June 2021. Secondly, in addition to the study of the convalescent patients, we tested the highest humoral responders following vaccination during convalescence (Figure 3A-D). Thirdly, we tested a panel of laboratory and health care workers that were one month post their third "booster dose" of BNT162b2 (Figure 3E). In each setting, the peak responses were tested across all relevant VOCs, Beta, Gamma, Delta, and Omicron. Finally, we tested five polyclonal human hyperimmune IgG batches that constitute pools of thousands of primarily US plasma donors collected around the peak of US vaccination (Figure 3F). This latter analysis establishes the extent of immune evasion at the population level, as the lgG is comprised of all plasma donors irrespective if they are convalescent and/or vaccinated. Finally, we tested therapeutic monoclonal antibodies including Sotrovimab, Casirivimab, Imdevimab, Bamlanivimab, Cilgavimab and Tixagevimab.

\section{Results And Discussion}

\section{Platform for rapid isolation and characterisation of humoral evasion in Omicron and other contemporary SARS-CoV-2 variants.}

Prior to June 2021, in the majority of Australian states, the only appearance of the SARS-CoV-2 variants was primarily through quarantine networks from returning overseas travellers ${ }^{4-6}$. New South Wales (NSW), over this time welcomed the majority of returning travellers entering Australia and during the period of December through to June provided a snapshot of globally circulating variants. Over the months of January to May in 2021, all VOCs and the majority of Variants Under Investigation (VUI) were detected through rapid whole genome sequencing (WGS) ${ }^{7-9}$ in the NSW quarantine networks. Access to this network through research-diagnostics partnerships, enabled the study of emerging variants shortly following diagnosis at a time when community spread in Australia was minimal. In this setting it provided a sentinel program, that had the potential to provide observations of the risk of key variants whilst individuals were isolated in quarantine. Whilst this continued largely for early 2021 , in June the Delta variant spread rapidly within the community and by the end of 2021 the Omicron variant had also started to spread alongside Delta. In quarantine networks, accessing variants was often restricted to a single sample and as such the ability to isolate current and emerging contemporary variants was paramount. Previously, we had developed a hyper-permissive cell line to SARS-CoV-2 based on the genetic engineering and screening of a HEK293T line (HAT-24) ${ }^{3}$. The use of this cell enabled virus isolation from greater than $80 \%$ of PCR-positive swabs, with all VOCs (Alpha, Beta, Gamma and Delta) and 6 VUls (Kappa, Eta, Zeta, Epsilon, lota and Lambda) isolated successfully in 2021. On the 29th of November, the first four positive cases of Omicron were detected in previously vaccinated asymptomatic 
returning travellers arriving from South Africa. Unlike VeroE6-derived cell lines, the HAT-24 cell line succumbs to rapid and extensive cytopathic effects ranging from plaque formation to extensive cell-cell fusion upon exposure to SARS-CoV-2, and for Omicron cytopathic effects were similar to that of previous SARS-CoV-2 variants (Figure 1B-D). In this setting, scoring of end-point titres of the four primary Omicron positive nasopharyngeal swabs was within range of other titres per diagnostic PCR value but lower than that observed in Delta primary samples (Figure 1A). It must be noted that the Delta samples were from primarily unvaccinated patient samples where in contrast Omicron samples were all from vaccinated patient samples. Over the same culture period, we observed no cytopathic effects using the VeroE6TMPRSS2 cell line over a four-day culture period.

Previously our attempts to use the HAT-24 line to perform antibody neutralisations was confounded by the increased permissiveness of this cell line to SARS-CoV- 2 infection over a prolonged culture period ${ }^{3}$. To limit the sensitivity of this cell line and also increase the utility of its use, we restricted assays to a 20hour time course. In this setting, we prelabelled cells with a live nucleic acid dye and then enumerated nuclei using high content microscopy and machine scoring 20 hours post-infection. As cytopathic effects generated large syncytia, this significantly reduced cell nuclei counts in a dose dependent manner $\left(R^{2}>\right.$ 0.99 across all variants tested) (Figure 1E-F). The introduction of neutralising serum then rescued cells from syncytia and increased cell counts in a dose dependent manner $\left(R^{2}>0.99\right)$ (Figure 2). We assessed the viral responses to the WHO International Standard and Reference Panel for anti-SARS-CoV-2 antibody 10 (Figure 2A-C) in addition to the Plasma Alliance (https://www.cslbehring.com/newsroom/2020/covig19-plasma-alliance-expands-membership) (Figure 2D-F) internal serology standard in HAT-24 cells versus VeroE6 cells. In initial tests we did not observe Omicron reach end-point neutralisation titres in either the HAT-24 or VeroE6 cell line. However, when observing the neutralisation titres of all other live SARS-CoV-2 variants that predated Omicron, we observed not only a strong correlation across variants $r=0.87$; $<<0.001$; Figure 2) but importantly equivalent neutralisation potency of the WHO international reference standard against the earlier B1 clade virus (neutralisation titre of 632 for the HAT-24 line versus 683 for the mean of all laboratories reporting to the $\mathrm{WHO}$ on live virus neutralisation assays ${ }^{10}$ ).

Using the above approach, not only were we able to isolate Omicron from all primary samples, but importantly we were able to expand virus, titre and determine neutralisation titres across many key serum samples and therapeutics within 7 days. Subsequent independent repeats led to consolidated datasets which were then communicated to both NSW and Australian Chief Health Officers to highlight the fold evasion of Omicron and what clinical therapeutics were available to treat cases within Australia as part of the COVID-19 response.

\section{Humoral evasion for Omicron relative to VOCs Beta, Gamma and Delta}

Initial tests on Omicron using peak serological responses (one month post-second dose) following two doses of BNT162b2 or ChAdOx1 nCoV-19 vaccines only reached end-point titres in 4 out of 17 donors tested (Supplementary Table S1). In order to determine titre reductions to statistical significance, we 
selected high neutralisation titre serum samples from the Australian ADAPT cohort (source of serum summarised in schematic from Figure 3). Neutralisations performed with convalescent sera from early clade infections (Figure 4A-B; Supplementary Figure 2A-B) showed an average of 4.7-fold reduction for Beta, 1.5-fold for Delta and 2.2-fold for Gamma relative to the ancestral strain (A.2.2). All responses observed with Omicron were below the limit of detection of our assay (serum dilution 1:20). For convalescent sera obtained from Delta wave infections (Figure 4C; Supplementary Figure 2C) we observed a mean ID50 of 52.6 for Omicron compared with mean ID50 values of 770.5 for the ancestral strain, 211.1 for Beta, 317.5 for Gamma and 556.5 for Delta (Supplementary Table S2). Compared to the ancestral strain this resulted in a 17.7-fold reduction in neutralisation with Omicron $(p<0.0001)$ compared to 4.0-fold for Beta ( $p<0.0024), 1.6$-fold for Delta and 2.9-fold for Gamma $(p<0.0365)$.

We next examined neutralisation responses in sera from convalescent donors from early clade infections who had subsequently been vaccinated with either the BNT162b2 or ChAdOx1 nCoV-19 vaccine (Figure 4D-E, Supplementary Figure 2D and Supplementary Table S2). While the vaccinated individuals showed a significant increase in neutralisation titres to the ancestral strain compared to convalescent donors (Supplementary Table S2), we again observed a 17.9 to 26.6 -fold reduction in neutralisation against Omicron ( $p<0.0001)$ compared to 3.7 to 4.1 -fold decrease observed for Beta $(p<0.0028)$. Similar results were observed with laboratory and health care worker $(\mathrm{HCW})$ volunteers at the peak of their third vaccine dose with BNT162b2, with a 16.9-fold reduction in neutralisation against Omicron $(p<0.0001)$ compared to a 4.4-fold reduction for Beta $(\mathrm{p}<0.0001$ ) (Figure 4F, Supplementary Figure 2F and Supplementary Table S3). Interestingly, there was a trend that vaccinated convalescent individuals had a greater fold reduction to Omicron compared with individuals who had received three BNT162b2 doses (20.2-fold versus 16.9fold), but this was not significant ( $P=0.12$, likelihood ratio test).

We obtained similar results when we tested neutralisation responses against five polyclonal human IgG batches comprising of more than ten thousand pooled plasma donors collected during the peak of the US vaccine rollout (Figure $4 \mathrm{G}$ and Supplementary Figure $2 \mathrm{G}$ ). There was a 16.8-fold reduction in neutralisation against Omicron $(p<0.0001)$ compared with 3.3-fold decrease for Beta $(p=0.0437)$. Similar fold reductions were also observed from polyclonal IgG that was collected from convalescent donors between September and October 2020 (fold reduction of 20.1-fold for Omicron versus 5.5-fold for Beta).

\section{Estimated fold reduction of Omicron and implications for vaccine efficacy}

We next estimated the fold reduction in neutralisation for each variant within each cohort (with censoring). To do so, we grouped the data into three convalescent groups (Conv1-First wave; Conv2Second wave; Conv3-Third wave (VOC Delta) see A, B and C in Figure 3), convalescent plus vaccinated, vaccine boosted (third dose), pooled polyclonal IgG and the WHO International Reference Standard as a control. This data is summarised in Figure 5. Note that Conv1 and Conv2 cohorts had no detectable neutralisation against Omicron and thus were completely excluded from this aggregated analysis. For estimating the reduction in vaccine efficacy, Conv3 individuals were also excluded since they were exposed to the Delta Spike immunogen and may have a different cross-reactivity (though including this 
group only changes the results very slightly). Since the fold-drop in neutralisation against Omicron was not significantly different in vaccinated convalescent individuals and boosted individuals, we grouped these individuals and found the average fold reduction across these groups was 17.3 -fold ( $95 \% \mathrm{Cl}$ : 15.0 20.0). Using this value, and the efficacy curve in Khoury et al ${ }^{11}$ we estimated the efficacy and confidence intervals for BNT162b2-vaccinated or boosted individuals (in the first few months after vaccination) (Table 1).

\section{Table 1: Estimates of Efficacy ( $95 \% \mathrm{Cls})$}

\begin{tabular}{|lll|}
\hline \multicolumn{3}{|c|}{ With censoring } \\
\cline { 2 - 3 } & BNT162b2 & mRNA boosted \\
\hline Symptomatic & $40.8 \%$ & $77.3 \%$ \\
& $(25.9 \%-55.0 \%)$ & $(65.1 \%-86.1 \%)$ \\
\hline Severe* & $81.4 \%$ & $96.4 \%$ \\
& $(50.9 \%-94.8 \%)$ & $(84.8 \%-99.3 \%)$ \\
\hline
\end{tabular}

*Note that Vaccine Efficacy (VE) against severe outcomes is much less well validated as there is insufficient data available with which to parameterise the model at such low titres against severe outcomes, in addition to a lack of understanding of baseline severity with Omicron.

\section{Modelling on vaccine efficacy based on Omicron fold evasion from humoral responses}

In combining the data obtained from the 50 patients with the highest neutralisation titres in the ADAPT cohort with the population humoral snapshot using polyclonal IgG from pooled plasma donors, it is evident that the fold reduction in neutralisation of Omicron was similar. Given the starting neutralisation level of the average BNT162b2 vaccinee against the ancestral virus (2.4-fold of convalescent individuals) ${ }^{11}$, a 17.3-fold drop brings the mean neutralisation level below the $50 \%$ protective level against symptomatic infection with Omicron. This neutralisation level corresponds to a predicted vaccine efficacy for otherwise naïve BNT162b2 vaccinated individuals of $40.8 \%(95 \% \mathrm{Cl}=25.9-55.0 \%)$ against symptomatic infection with Omicron, and $77.3 \%(95 \% \mathrm{Cl}=65.1 \%-86.1 \%)$ protection against severe infection (Table 1), in the first months after vaccination. Previously we have shown that mRNA vaccination of previously infected individuals produce neutralisation titres that are significantly higher than observed in current two-dose vaccination regimes ${ }^{12}$. Thus, even with the 17.3-fold decrease in neutralisation titre, boosting with mRNA vaccines is predicted to provide significant protection from infection with Omicron (Table 1). 


\section{$\mathrm{IC}_{50}(\mathrm{ng} / \mathrm{mL})$}

\begin{tabular}{|c|c|c|c|c|}
\hline $\mathrm{mAb}$ & Developer & $\begin{array}{l}\text { Ancestral } \\
\text { (A.2.2) }\end{array}$ & $\begin{array}{l}\text { Omicron } \\
\text { (B.1.1.529) }\end{array}$ & $\begin{array}{l}\text { Fold- } \\
\text { change }\end{array}$ \\
\hline Sotrovimab & Vir Biotechnology / GSK & 372 & 1059 & 2.8 \\
\hline Casirivimab & Regeneron & 27 & $\mathrm{nn}($ to $1 \mu \mathrm{g} / \mathrm{mL}$ ) & $\mathrm{N} / \mathrm{A}$ \\
\hline Imdevimab & Regeneron & 25 & $\mathrm{nn}$ (to $1 \mu \mathrm{g} / \mathrm{mL}$ ) & $\mathrm{N} / \mathrm{A}$ \\
\hline Bamlanivimab & $\begin{array}{l}\text { AbCellera Biologics / Eli } \\
\text { Lilly }\end{array}$ & 32 & $\mathrm{nn}$ (to $10 \mu \mathrm{g} / \mathrm{mL}$ ) & N/A \\
\hline Cilgavimab & Astra Zeneca & 18 & $\mathrm{nn}$ (to $1 \mu \mathrm{g} / \mathrm{mL}$ ) & N/A \\
\hline Tixagevimab & Astra Zeneca & 47 & 3490 & 73.8 \\
\hline Ab-3467 & Burnett et al. ${ }^{13}$ & 502 & $\mathrm{nn}$ (to $10 \mu \mathrm{g} / \mathrm{mL}$ ) & $\mathrm{N} / \mathrm{A}$ \\
\hline
\end{tabular}

Table 2: Neutralisation of SARS-CoV-2 Omicron variant by commercially developed monoclonal antibodies and the class 4 Ab-3467.

The Omicron variant contains several mutations at RBD sites, previously thought to be highly conserved, that are the target of antibody therapeutics. For example, S371L, S373P, and S375F form part of the class 4 epitope, affecting previously described class 4 antibodies such as Ab-3467 that broadly neutralise sarbecoviruses ${ }^{13,14}$. Additional mutations unique to Omicron at sites $417,440,446$, and 493 are likely to contribute to the lack of the neutralisation of other therapeutic antibodies. The class 3 antibody Sotrovimab targets a highly conserved region of sarbecovirus RBD ${ }^{15}$ and retains neutralising activity against Omicron in live virus neutralisation, with two mutations within its epitope (G339D and N440K) likely resulting in only a moderate reduction in potency observed against Omicron relative to the ancestral SARS-CoV-2 lineage. As new variants of SARS-CoV-2 emerge that have altered transmissibility and disease phenotype, the availability of therapeutic and prophylactic mAbs that remain broadly active is essential. Although the retention of neutralising activity by Sotrovimab against Omicron is promising, the complete loss of activity of many other monoclonals remains a concern, and the development of new and improved monoclonal antibody modalities is urgently warranted.

To conclude, Omicron represents a significant challenge to the existing two dose vaccination strategy presently adopted by many countries globally. Whilst the VOCs Beta and Gamma also represented challenges to vaccine efficacy, there are two defining features of Omicron that provide additional concerns. Firstly, as observed herein the fold evasion to humoral immunity is significantly greater with Omicron than all other VOCs. Secondly, unlike Beta and Gamma, Omicron is gaining momentum in global prevalence in areas where Delta dominated in late 2021. Whilst boosters utilising the same Clade A Spike increase antibody titre to Omicron, development of either variant specific boosters or vaccine 
formulations that enable greater breadth will be more pragmatic in the longer term. The latter will be very important in those groups that may have a limited titre, such as in the elderly or immunocompromised. Fortunately for the latter at risk groups, certain immunotherapeutic treatments like Sotrovimab appear to maintain potency and remain relevant for treatment in Omicron cases.

\section{Materials And Methods}

\section{Human sera and ethics statement}

The Adapting to Pandemic Threats (ADAPT) cohort is composed of RT-PCR-confirmed convalescent individuals (including some subsequently vaccinated) recruited in Australia since $2020^{3}$. Serum from healthy volunteers vaccinated with ChAdOx1 nCoV-19 and BNT162b2 was collected 4 weeks post second-dose vaccination. A subgroup of ADAPT participants with the highest neutralisation responses were selected for this study (Supplementary Table S2). Sera from 25 healthy laboratory and health care workers (HCW) were collected at 1 month post-third vaccine dose with BNT162b2 and were used to study vaccine booster responses against virus variants. All human serum samples were obtained with written informed consent from the participants (2020/ETH00964; 2020/ETH02068; 2019/ETH03336; 2021/ETH00180).

\section{Other immunoglobulin products}

For monoclonal antibodies, DNA sequences encoding the variable domain sequences of the therapeutic monoclonal antibodies Sotrovimab, Casirivimab, Imdevimab, Bamlanivimab, Cilgavimab and Tixagevimab were generated by gene synthesis, cloned into human IgG1 expression vectors, and produced in $\mathrm{CHO}$ cells ${ }^{13}$.

\section{Polyclonal Immunoglobulin preparations and anti-SARS-CoV-2 hyperimmune globulin}

A CoVlg-19 Plasma Alliance (Poly IgG Convalescent 869) was formed in 2020 between major plasma pharmaceuticals including CSL, Takeda, Octapharma and Sanquin with an aim to develop a COVID-19 immunoglobulin therapy. As part of that initiative, CSL Behring manufactured anti-SARS-CoV-2 hyperimmune globulin (CoVlg). Approximately 5000 convalescent donor plasma units were collected between September and October 2020, exclusively from SARS-CoV-2 convalescent donors after COVID-19 confirmation ${ }^{16}$, the immunoglobulin purified using the licensed and fully validated immunoglobulin manufacturing process used for Privigen ${ }^{17}$, notionally similar to others ${ }^{18}$. Five IVIG lots (Poly IgG 1033, $4850,7450,0301,0723$ ) manufactured using the Privigen process described by Stucki et al. ${ }^{17}$ included US plasma collected by plasmapheresis from a mixture of vaccinated with SARS-CoV-2 mRNA vaccines, convalescent and non-convalescent donors (source plasma, $n$ between 9495-23,667 per batch) majority of donations collected between April and June 2021. The WHO international reference standard for SARSCoV-2 neutralisation (NIBSC 20/136) was obtained from ${ }^{10}$. 
HEK293T cells (Thermofisher Scientific, \#R70007) stably expressing human ACE2 and TMPRSS2 were generated by lentiviral transductions as previously described ${ }^{3}$. A highly permissive clone (HAT-24) was identified through clonal selection and used for this study. The HAT-24 cell line has been extensively cross-validated with the VeroE6 cell line using several hundred serum samples derived from the ADAPT cohort. The results of the WHO Alliance serology standards presented herein summarise the crossvalidation results between the HAT-24 and VeroE6 cell line. HAT-24 cells and VeroE6-TMPRSS2 cells (CellBank Australia, JCRB1819) were cultured in DMEM-10\%FBS and VeroE6 cells (ATCC $\AA$ CRL-1586 ${ }^{\text {TM}}$ ) in MEM- $10 \%$ FBS. All cells were incubated at $37^{\circ} \mathrm{C}, 5 \% \mathrm{CO}_{2}$ and $>90 \%$ relative humidity.

\section{Viral isolation, propagation, and titration}

All laboratory work involving infectious SARS-CoV-2 occurred under biosafety level 3 (BSL-3) conditions. Diagnostic respiratory specimens testing positive for SARS-CoV-2 (RT-qPCR, Seegene Allplex SARS-CoV2) were sterile-filtered through $0.22 \mu \mathrm{m}$ column-filters at 10,000 $\mathrm{xg}$ and serially diluted (1:3) on HAT-24 cells ( $10^{4}$ cells/well in 96-well plates). Upon confirmation of cytopathic effect in the HAT-24 line by light microscopy, $300 \mu \mathrm{L}$ pooled culture supernatant from infected wells (passage 1) were added to VeroE6 cells in a 6-well plate $\left(0.5 \times 10^{6}\right.$ cells/well in $\left.2 \mathrm{~mL}\right)$ and incubated for 48 hours. The supernatant was cleared by centrifugation ( $2000 \mathrm{xg}$ for 5 minutes), frozen at $-80^{\circ} \mathrm{C}$ (passage 2 ), then thawed and titrated to determine median tissue culture infective dose (TCID50) on VeroE6 or VeroE6-TMPRSS2 cells according to the Spearman-Karber method ${ }^{19}$. Viral stocks used in this study correspond to passage 3 virus, which were generated by infecting the VeroE6-TMPRSS2 cells at MOI $=0.025$ for all variants with the exception of Omicron. For the latter Omicron was expanded only in the VeroE6 line, as lower titres were observed when expanding virus using the VeroE6-TMPRSS2 line. For all variants, passage 3 expansions were on for 24 hours before collecting, clearing, and freezing the supernatant as above. This ensures a similar infectivity to particle ratio across all variants. Sequence identity and integrity were confirmed for both passage 1 and passage 3 virus via whole-genome viral sequencing using an amplicon-based Illumina sequencing approach, as previously described ${ }^{9}$. For a list of the viral variants used in this study see Supplementary Table S3. Passage 3 stocks were titrated by serial dilution (1:5) in DMEM-5\%FBS, mixing with HAT-24 cells live-stained with 5\% v/v nuclear dye (Invitrogen R37605) at $1.6 \times 10^{4} /$ well in 384-well plates, incubating for 20 hours, and determining whole-well nuclei counts with an InCell Analyzer highcontent microscope and IN Carta analysis software (Cytiva, USA). Data was normalised to generate sigmoidal dose-response curves (average counts for mock-infected controls $=100 \%$, and average counts for highest viral concentration $=0 \%$ ) and median lethal dose (LD50) values were obtained with GraphPad Prism software.

\section{Rapid high-content SARS-CoV-2 microneutralisation assay with HAT-24 cells (R20)}

Human sera or monoclonal antibodies were serially diluted (1:2 series starting at 1:10) in DMEM-5\%FBS and mixed in duplicate with an equal volume of SARS-CoV-2 virus solution standardised at 2xLD50. After 1 hour of virus-serum coincubation at $37^{\circ} \mathrm{C}, 40 \mu \mathrm{L}$ was added to an equal volume of nuclear-stained HAT-24 cells pre-plated in 384-well plates as above. Plates were incubated for 20 hours before 
enumerating nuclear counts with a high-content fluorescence microscopy system as indicated above. The $\%$ neutralisation was calculated with the formula: $\% \mathrm{~N}=(\mathrm{D}-(1-\mathrm{Q})) \times 100 / \mathrm{D}$ as previously described ${ }^{3}$.

Briefly, "Q" is a well's nuclei count divided by the average count for uninfected controls (defined as having $100 \%$ neutralisation) and $D=1-Q$ for the average count of positive infection controls (defined as having $0 \%$ neutralisation). Sigmoidal dose-response curves and ID50 values (reciprocal dilution at which $50 \%$ neutralisation is achieved) were obtained with GraphPad Prism software. Neutralisation assays with VeroE6 cells were performed exactly as described above excepting that; input virus solution was standardised at $1.25 \times 10^{4} \mathrm{TCID} 50 / \mathrm{mL}$, cells were seeded at $5 \times 10^{3}$ cells/well in MEM-2\%FBS (final MOI $=0.05$ ), plates were incubated for 72 hours, and cells were stained with nuclear dye only 1 hour before imaging.

\section{Estimating vaccine efficacy against Omicron}

Vaccine efficacy against Omicron was estimated using the approach and model previously reported 420 . Briefly, we estimate vaccine efficacy (VE) for a given vaccine/cohort against a given variant based on the $\left(\log _{10}\right)$ geometric mean (GM) neutralisation titre, normalised to the GM neutralisation titre (against ancestral virus) of convalescent individuals (exposed to ancestral virus). This normalised ( $\left.\log _{10}\right) \mathrm{GM}$ titre is given by, and the VE for a given is predicted using the equation

$$
V E(\mu)=\int_{-\infty}^{\infty} N(x, \mu, \sigma) \frac{1}{1+e^{-k\left(x-x_{50}\right)}} d x,
$$

where, $N$ is the probability density function of a normal distribution of the distribution in $\left(\log _{10}\right)$ neutralisation titres with mean $\mu$ and standard deviation $\sigma$, and $x_{50}$ and $k$ are the parameters of a logistic function representing the $\left(\log _{10}\right)$ normalised neutralisation titre associated with $50 \%$ protection and $k$ determines the steepness of the logistic function. The parameters $\sigma=0.46$ (representing the spread of neutralisation titres in vaccinated individuals), $x_{50}=\left(\log _{10}\right) 0.20$ and $k=3.1$ were estimated previously by fitting vaccine efficacy data from randomised control trials across 7 SARS-CoV-2 vaccines ${ }^{4}$. Thus, vaccine efficacy of BNT162b2 vaccinated individuals against Omicron is estimated by the normalised GM neutralisation titre of BNT162b2 vaccinated individuals against Omicron. Previously we have estimated that BNT162b2 vaccinated individuals have a GM neutralisation titre (against ancestral virus) that is 2.4-fold times the GM neutralisation titre of convalescent individuals (who were exposed to ancestral virus) ${ }^{11}$. Thus, to estimate the VE against Omicron of BNT162b2 vaccinated individuals, we calculate the normalised GM neutralisation titre of these individuals against Omicron. A 20.2-fold drop in neutralisation against Omicron is estimated to lead to a GM neutralisation titre in BNT162b2 vaccinated individuals against Omicron of -fold of the GM neutralisation titre of convalescent individuals (after exposure to ancestral virus) against ancestral virus (thus, $\mu=\left(\log _{10}\right) 0.119$ in the above equation to estimate vaccine efficacy for BNT162b2 vaccinated individuals against Omicron). Similar, we have previously shown that $\mathrm{mRNA}$ vaccines in previously infected individuals produce $\mathrm{GM}$ neutralisation titres that are approximately 12.0 -fold greater than the GM titre of convalescent individual who were exposed to 
ancestral virus $^{12}$. Thus, the 20.2 -fold loss of neutralisation to Omicron is estimated to give a GM neutralisation titre against Omicron that is 0.594 -fold of the GM of convalescent subjects against ancestral virus (after exposure to ancestral virus). Thus, to estimate vaccine efficacy for previously infected and mRNA vaccinated individuals we use $\mu=\left(\log _{10}\right) 0.594$ in the above equation. Confidence intervals for predicted vaccine efficacies were estimated using bootstrapping.

\section{Declarations}

Competing interests: The authors declare no competing interests.

\section{References}

1 Liu, Z. et al. Identification of SARS-CoV-2 spike mutations that attenuate monoclonal and serum antibody neutralization. Cell host \& microbe 29, 477-488 e474, doi:10.1016/j.chom.2021.01.014 (2021).

2 Zahradnik, J. et al. SARS-CoV-2 variant prediction and antiviral drug design are enabled by RBD in vitro evolution. Nat Microbio/ 6, 1188-1198, doi:10.1038/s41564-021-00954-4 (2021).

3 Tea, F. et al. SARS-CoV-2 neutralizing antibodies: Longevity, breadth, and evasion by emerging viral variants. PLoS Med 18, e1003656, doi:10.1371/journal.pmed.1003656 (2021).

4 Lane, C. R. et al. Genomics-informed responses in the elimination of COVID-19 in Victoria, Australia: an observational, genomic epidemiological study. Lancet Public Health 6, e547-e556, doi:10.1016/S2468-2667(21)00133-X (2021).

5 Leong, L. E. X. et al. Whole-Genome Sequencing of SARS-CoV-2 from Quarantine Hotel Outbreak. Emerg Infect Dis 27, 2219-2221, doi:10.3201/eid2708.204875 (2021).

6 Andersson, P., Sherry, N. L. \& Howden, B. P. Surveillance for SARS-CoV-2 variants of concern in the Australian context. Med J Aust 214, 500-502 e501, doi:10.5694/mja2.51105 (2021).

7 Seemann, T. et al. Tracking the COVID-19 pandemic in Australia using genomics. Nat Commun 11, 4376, doi:10.1038/s41467-020-18314-x (2020).

8 Rockett, R. J. et al. Revealing COVID-19 transmission in Australia by SARS-CoV-2 genome sequencing and agent-based modeling. Nat Med 26, 1398-1404, doi:10.1038/s41591-020-1000-7 (2020).

9 Bull, R. A. et al. Analytical validity of nanopore sequencing for rapid SARS-CoV-2 genome analysis. Nat Commun 11, 6272, doi:10.1038/s41467-020-20075-6 (2020).

10 Kristiansen, P. A. et al. WHO International Standard for anti-SARS-CoV-2 immunoglobulin. Lancet 397, 1347-1348, doi:10.1016/S0140-6736(21)00527-4 (2021). 
11 Khoury, D. S. et al. Neutralizing antibody levels are highly predictive of immune protection from symptomatic SARS-CoV-2 infection. Nat Med 27, 1205-1211, doi:10.1038/s41591-021-01377-8 (2021).

12 Cromer, D. et al. Neutralising antibody titres as predictors of protection against SARS-CoV-2 variants and the impact of boosting: a meta-analysis. The Lancet Microbe, doi:10.1016/S26665247(21)00267-6 (2021).

13 Burnett, D. L. et al. Immunizations with diverse sarbecovirus receptor-binding domains elicit SARSCoV-2 neutralizing antibodies against a conserved site of vulnerability. Immunity, doi:10.1016/j.immuni.2021.10.019 (2021).

14 Rouet, R. et al. Potent SARS-CoV-2 binding and neutralization through maturation of iconic SARSCoV-1 antibodies. MAbs 13, 1922134, doi:10.1080/19420862.2021.1922134 (2021).

15 Pinto, D. et al. Cross-neutralization of SARS-CoV-2 by a human monoclonal SARS-CoV antibody. Nature 583, 290-295, doi:10.1038/s41586-020-2349-y (2020).

16 Kober, C. et al. IgG3 and IgM Identified as Key to SARS-CoV-2 Neutralization in Convalescent Plasma Pools. PLoS One 17, e0262162, doi:10.1371/journal.pone.0262162 (2022).

17 Stucki, M. et al. Investigations of prion and virus safety of a new liquid IVIG product. Biologicals 36, 239-247, doi:10.1016/j.biologicals.2008.01.004 (2008).

18 Karbiener, M. et al. Plasma from post-COVID-19 and COVID-19-Vaccinated Donors Results in Highly Potent SARS-CoV-2 Neutralization by Intravenous Immunoglobulins. J Infect Dis, doi:10.1093/infdis/jiab482 (2021).

19 Ramakrishnan, M. A. Determination of $50 \%$ endpoint titer using a simple formula. World J Viro/ 5, 85-86, doi:10.5501/wjv.v5.i2.85 (2016).

20 Cromer, D. et al. Neutralising antibody titres as predictors of protection against SARS-CoV-2 variants and the impact of boosting: a meta-analysis. Lancet Microbe, doi:10.1016/S26665247(21)00267-6 (2021).

\section{Supplementary Table Captions}

Supplementary Table S1: End-point titres for two dose BNT162b2 or ChAdOx1 nCoV-19 vaccine recipients one month post-second dose.

Supplementary Table S2: ADAPT participants and ID50s.

Supplementary Table S3: Neutralisation assay on sera from healthy donors 1-month post-third vaccine dose. 
Supplementary Table S3: SARS-CoV-2 variants used in this study.

\begin{tabular}{llll} 
Variant & Lineage & Seq ID & GISAID ID \\
\hline Ancestral & A.2.2 & nCoV_2715 & hCoV-19/Australia/NSW4715/2021 \\
\hline Beta & B.1.351 & nCoV_2463 & hCoV-19/Australia/NSW4463/2021 \\
\hline Gamma & P.1 & nCoV_2319 & hCoV-19/Australia/NSW4318/2021 \\
\hline Delta & B.1.617.2 & nCoV_2605 & hCoV-19/Australia/NSW4605/2021 \\
\hline Omicron & B.1.1.529 & RCoV_1933 & hCoV-19/Australia/NSW-RPAH-1933/2021
\end{tabular}

Figures 

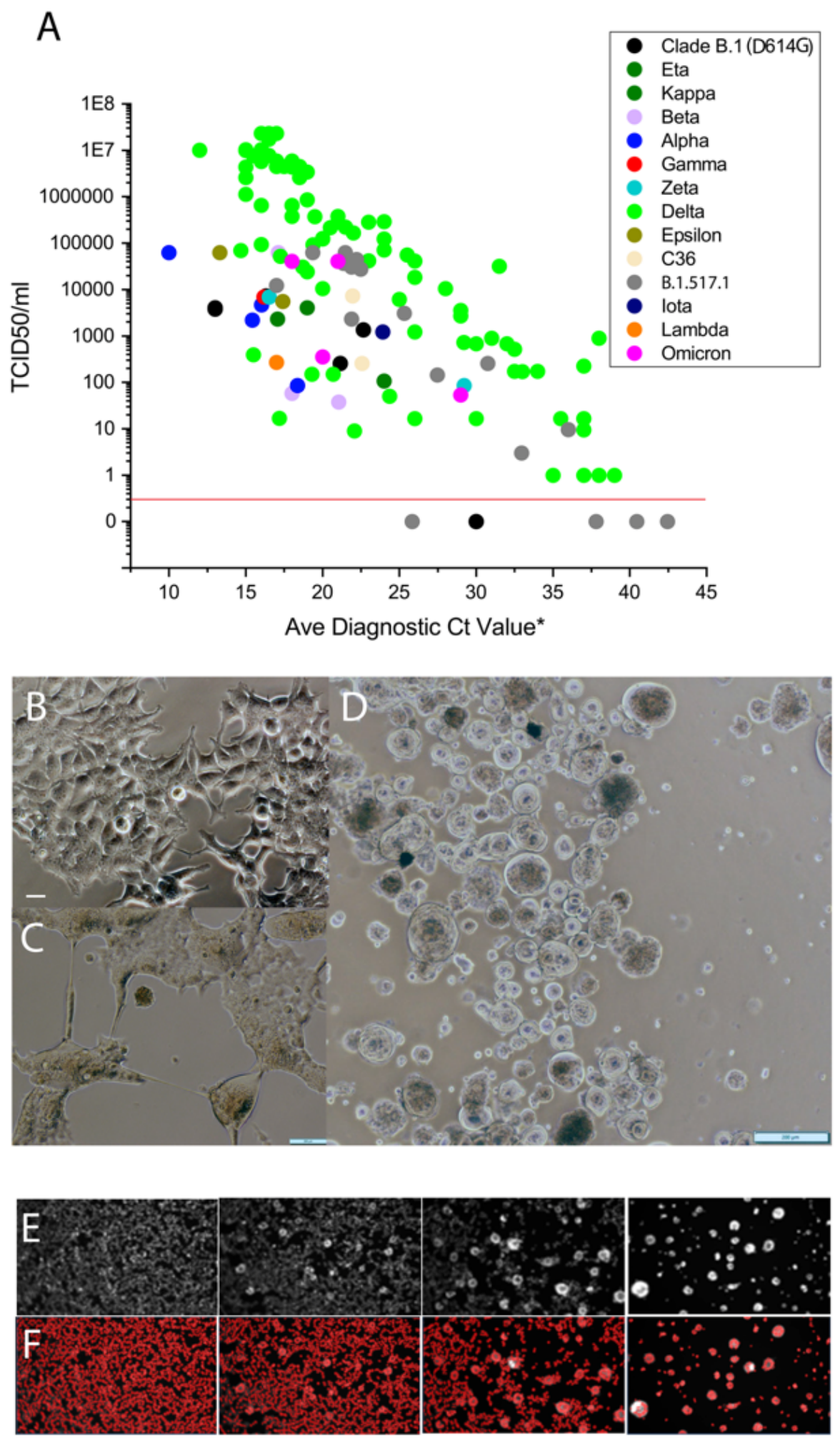

Figure 1

Isolation of Omicron versus all SARS-CoV-2 VOC and key VUI in 2021. 
A

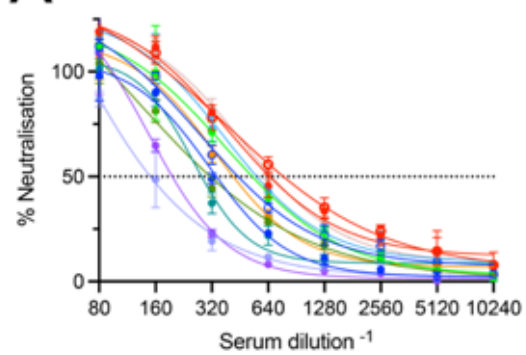

D

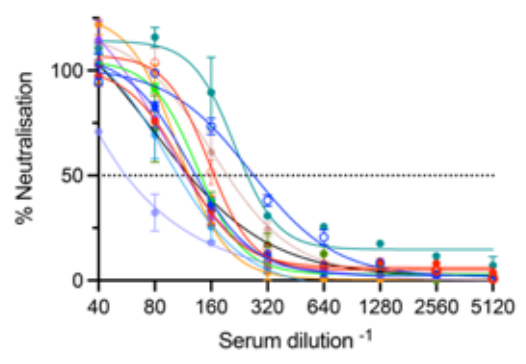

\begin{tabular}{|c|c|}
\hline$-\mathrm{A} .2 .2$ & - Epsilon (B.1.429) \\
\hline- B.1.2 & - Zeta (P.2) \\
\hline$\bullet-$ Alpha (B.1.1.7) & $-\bullet-E t a(B .1 .525)$ \\
\hline- Beta (B.1.351) & $\rightarrow-$ Kappa (B.1.617.1) \\
\hline$-\bullet-G a m m a(P .1)$ & - - Lambda (C.37) \\
\hline -๑-Delta (B.1.617.2) & $-\mathrm{C} .36$ \\
\hline
\end{tabular}

B

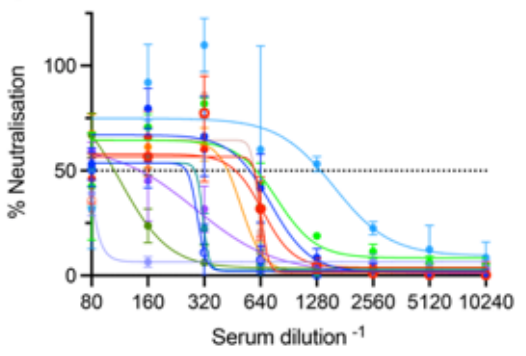

$\mathrm{E}$

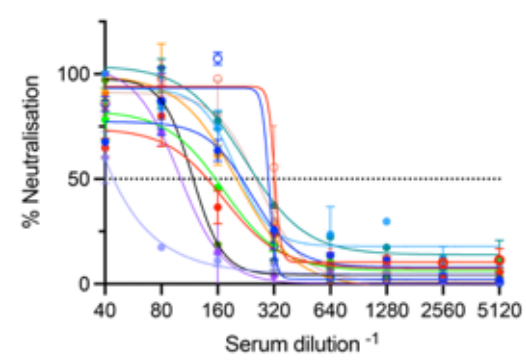

C

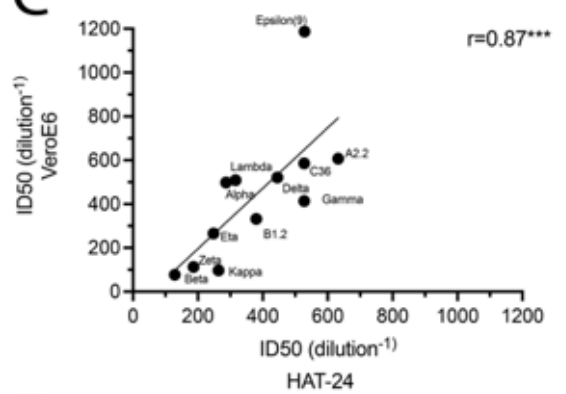

$\mathrm{F}$

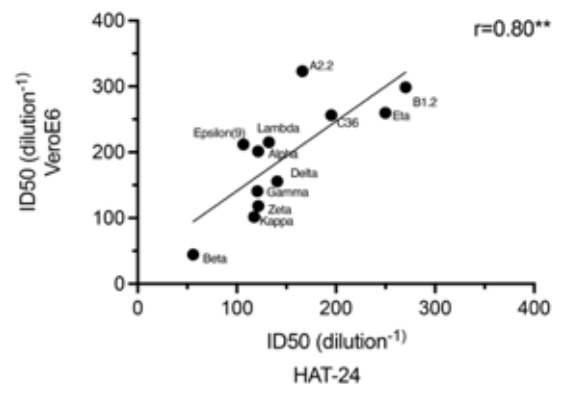

Figure 2

Cross-validation of HAT-24 cells against VeroE6 cell line utilising the WHO international reference standard $\mathrm{G}$ and Plasma Alliance Standard with 12 live primary SARS-CoV-2 viruses. 
Australian convalescent donors \& SARS-CoV-2 infection waves: ADAPT cohort

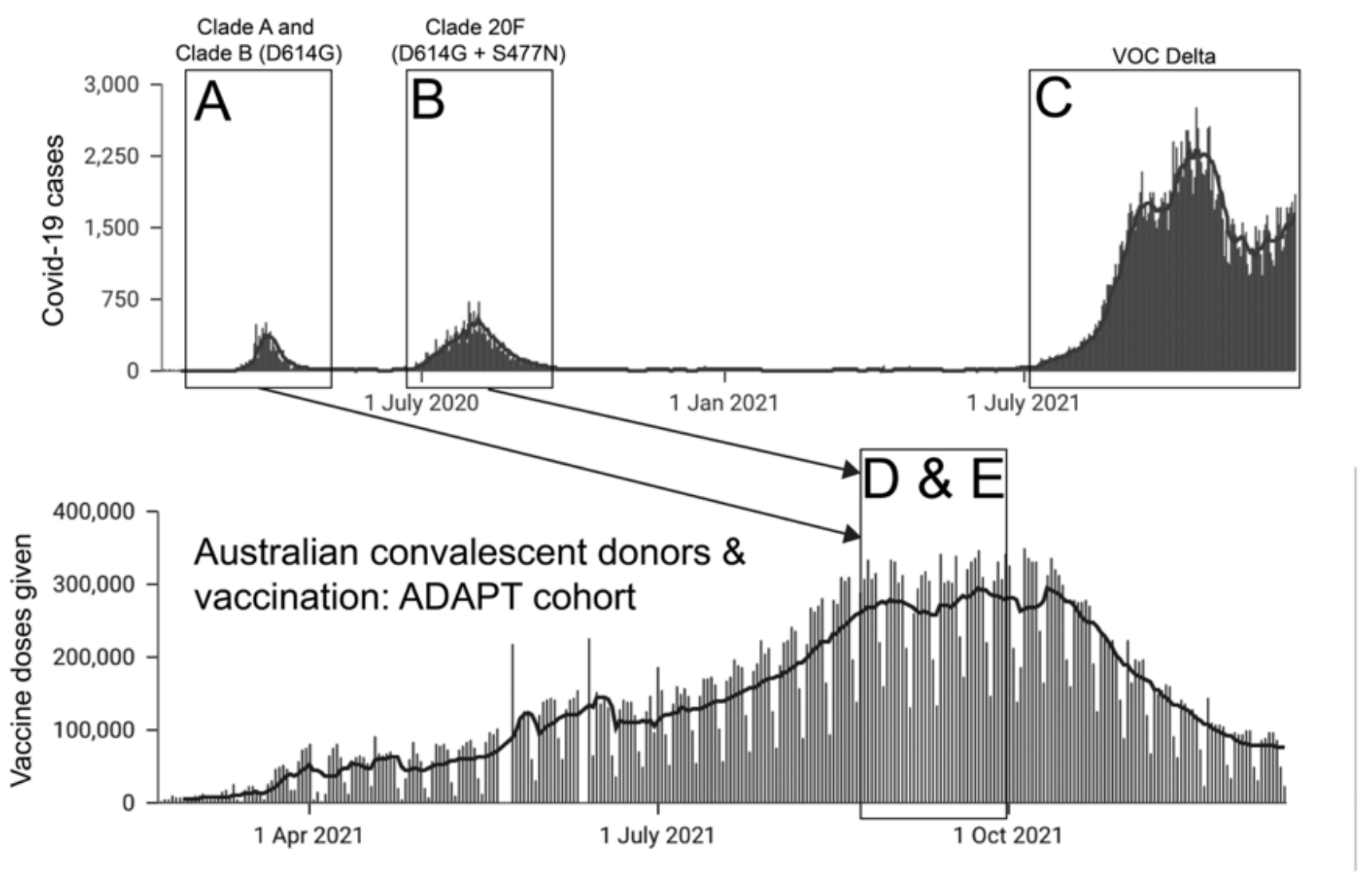

Concentrated US plasma donor IgG

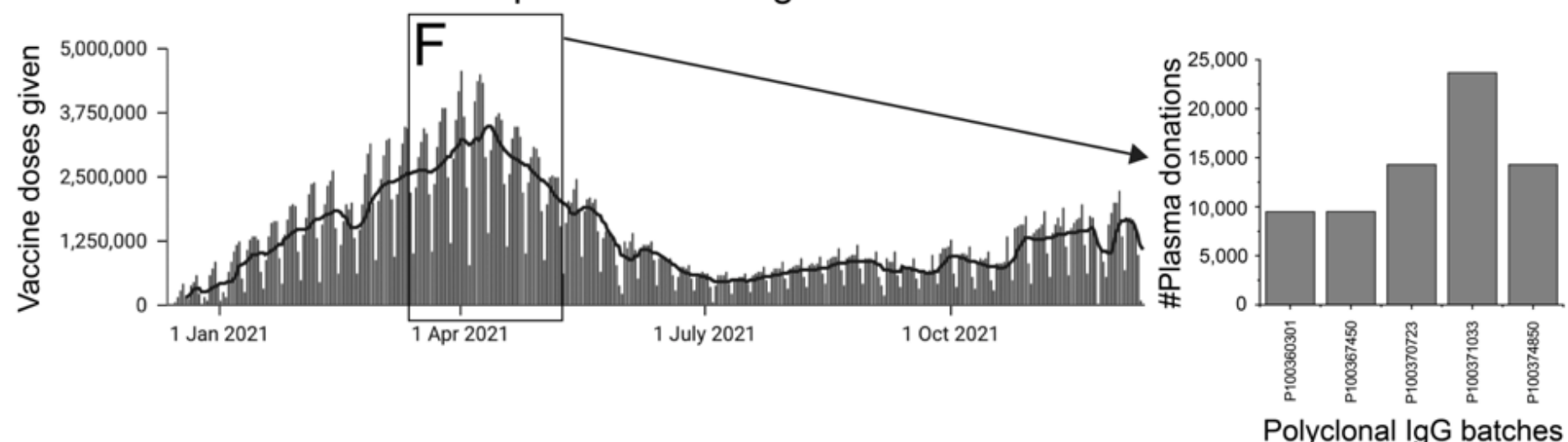

Figure 3

Source of high titre serology samples and polyclonal IgG obtained during the COVID-19 pandemic. 
A

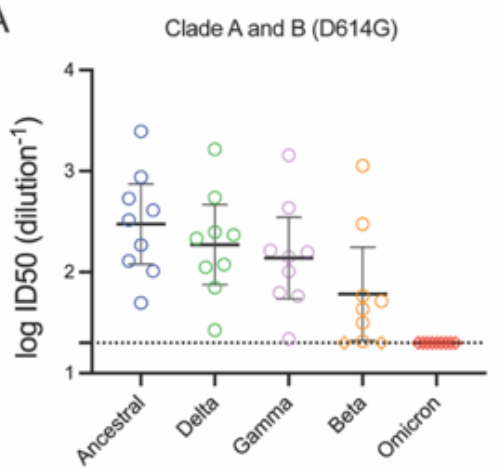

C

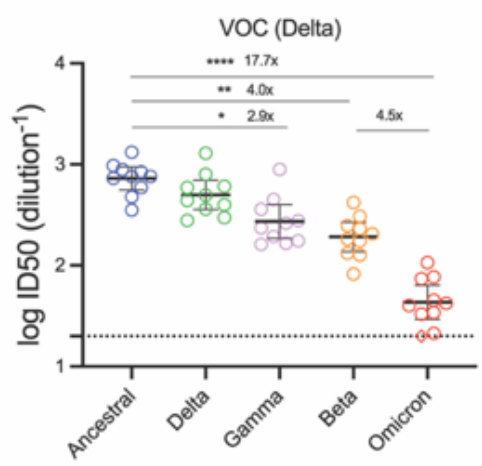

E

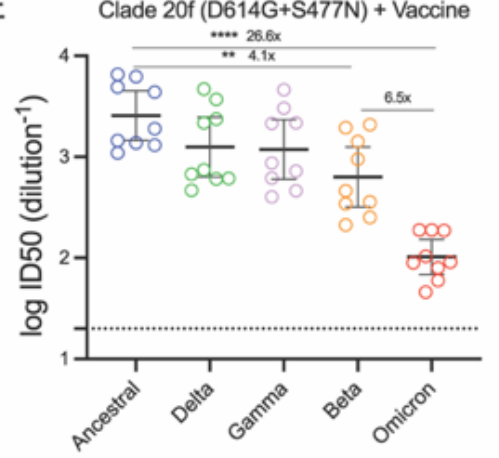

G

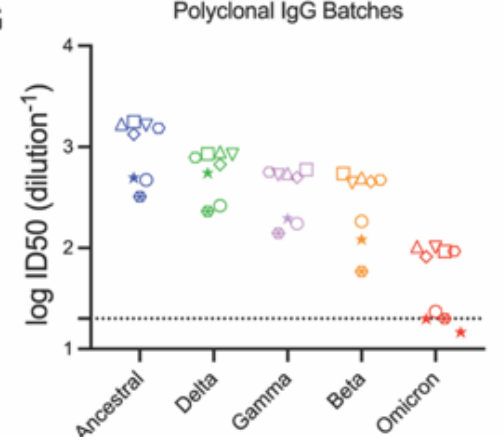

B

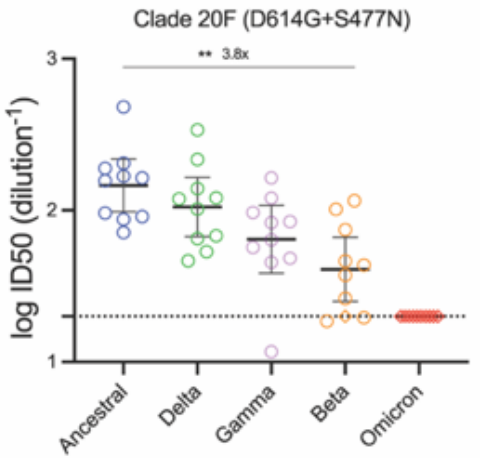

D

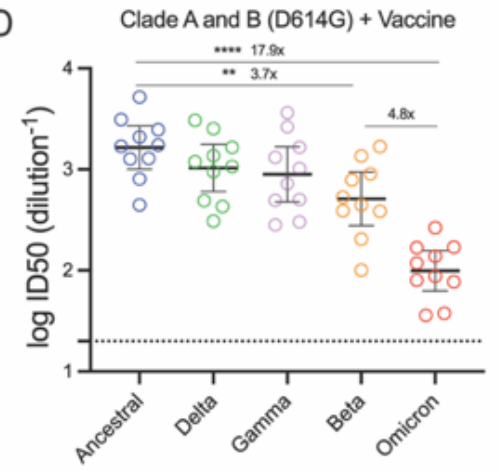

F

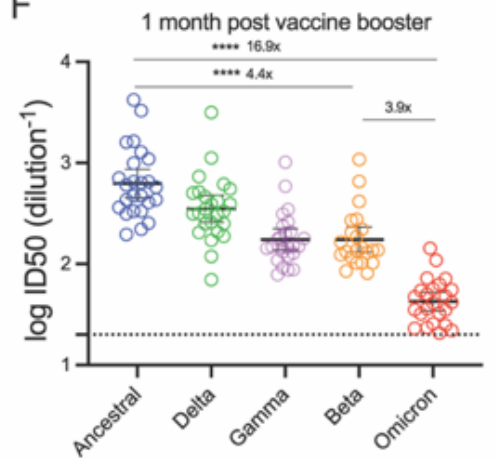

\section{Figure 4}

Humoral neutralisation of live SARS-CoV-2 variants in convalescent and vaccinated donors and concentrated human Ig $\underline{\text { plasma samples. }}$ 


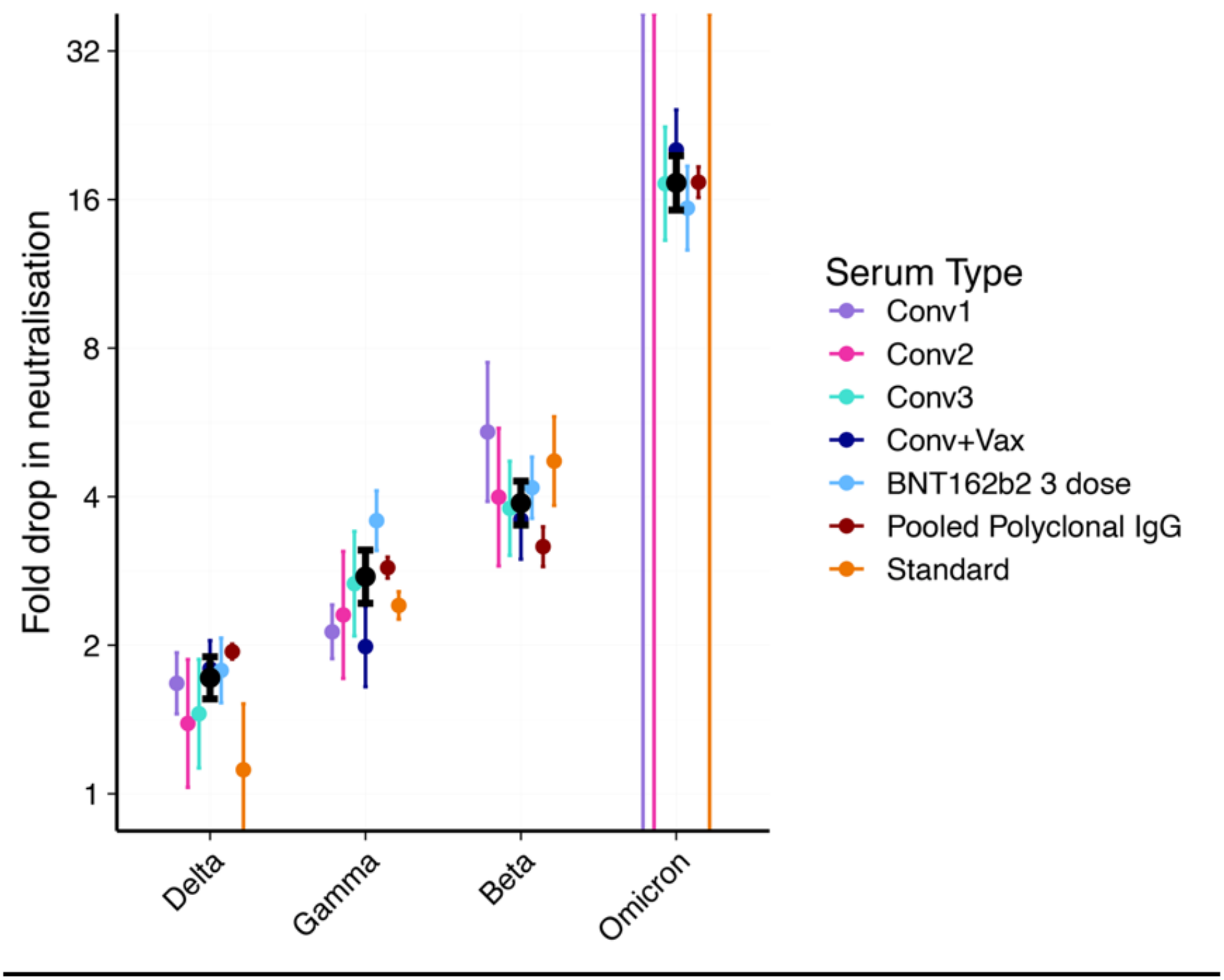

Figure 5

Summary of neutralisation reductions across Omicron and other variants of concern.

\section{Supplementary Files}

This is a list of supplementary files associated with this preprint. Click to download.

- Supplementarytable1.xlsx

- SupplementarytableS1.xlsx

- SupplementarytableS2.xlsx

- SupplementarytableS3.xlsx

- SupplementaryFigure1.docx 